\title{
Article \\ Effect of Sn and Mo on Microstructure and Electrochemical Property of TiZrTaNb High Entropy Alloys
}

\author{
Qiaoyu Li, Tengfei Ma *, Yuliang Jin, Xiaohong Wang *, Duo Dong and Dongdong Zhu
}

Citation: Li, Q.; Ma, T.; Jin, Y.; Wang, X.; Dong, D.; Zhu, D. Effect of Sn and Mo on Microstructure and

Electrochemical Property of TiZrTaNb High Entropy Alloys. Crystals 2021, 11, 1527. https://doi.org/10.3390/ cryst11121527

Academic Editors: Sergio Brutti, Geun Woo Lee and Hyokyung Sung

Received: 4 November 2021

Accepted: 5 December 2021

Published: 7 December 2021

Publisher's Note: MDPI stays neutral with regard to jurisdictional claims in published maps and institutional affiliations.

Copyright: (c) 2021 by the authors. Licensee MDPI, Basel, Switzerland. This article is an open access article distributed under the terms and conditions of the Creative Commons Attribution (CC BY) license (https:// creativecommons.org/licenses/by/ $4.0 /)$.
Key Laboratory of Air-Driven Equipment Technology of Zhejiang Province, Quzhou University, Quzhou 324000, China; L2289136524@163.com (Q.L.); J17625852076@163.com (Y.J.); 36077@qzc.edu.cn (D.D.); 36073@qzc.edu.cn (D.Z.)

* Correspondence: 36105@qzc.edu.cn (T.M.); 36098@qzc.edu.cn (X.W.); Tel.: +86-18191269298 (T.M.)

Keywords: high entropy alloys; TiZrTaNb alloy; microstructure; electrochemical property

\section{Introduction}

A high entropy alloy is a new alloy proposed at the beginning of this century [1-3]. It is a multiprincipal element alloy with five or more main elements and each element's content is between $5 \%$ and $35 \%$, which breaks the design concept of the traditional alloy and creates a new idea for alloy design [4-6]. The unique composition characteristics of the high entropy alloy lead to excellent properties such as high strength, high wear resistance and excellent corrosion resistance compared to traditional alloys, showing wide application prospects in aerospace, nuclear power and biomedical fields [7-11]. Previous studies have shown that the elastic modulus of a high-entropy alloy with BCC structure is low, which can achieve a better match with the human body and is more suitable for implantation materials [12-14]. Todai et al. prepared a series of high-entropy alloys for use in bone repair (TiNbTaZrHf, TiNbTaZr, TiNbTaZrMo, etc.) by alloying safe elements without cytotoxicity and sensitization factors. The as-cast TiZrTaNb exhibited a single BCC phase and achieved a compressive yield strength of $1100 \pm 90 \mathrm{MPa}$, strain of $48 \pm 6 \%$, and modulus of $116 \pm 6 \mathrm{GPa}$ [15]. Moreover, the mechanical properties were improved after the addition of Mo element, which showed better biocompatibility than pure titanium and stainless steel [16-18]. The effect of elements can not only affect the mechanical properties, but also the electrochemical property which is a key parameter for biological materials [19-21]. Alloying designation is still a hot topic in material research, especially for the high entropy alloy, due to the complex element interactions.

The Sn element is commonly used in Ti-based biological alloy, which has non-toxic effects. In this paper, the effects of $\mathrm{Sn}$ and Mo elements on a TiZrNbTa high entropy alloy were studied, and three high entropy alloys (TiZrTaNb, TiZrTaNbMo and TiZrTaNbSn) with equal atomic ratio were designed. The effects of alloying elements on microstructure 
and properties were studied by means of scanning electron microscope (SEM), X-ray diffractometer (XRD) and electrochemical workstation.

\section{Materials and Methods}

High purity metal particles $\mathrm{Ti}, \mathrm{Zr}, \mathrm{Ta}, \mathrm{Nb}, \mathrm{Mo}$ and $\mathrm{Sn}$ (purity $\geq 99.9 \%$ ) were selected as raw materials(Tim(Beijing)New Material Technology Co.,LTD), and arc melting was carried out in a high vacuum arc melting furnace (Model DHL-500LL) (The origin is Shenyang, China, purchased from Shenyang Scientific Instrument Co., Ltd. of the Chinese Academy of Sciences) to obtain high entropy alloy ingots. Three high entropy alloys are TiZrTaNb, TiZrTaNbMo and TiZrTaNbSn (at. \%). The high melting point metal was placed at the top of crucible and low melting point metal was placed at the bottom, and then different melting point metals can be completely melted. The ingot was remelted five times and homogeneous chemical composition was achieved. No wrinkles and black pits were observed on the surface of ingot.

The electrochemical corrosion performance was tested on the CHI604E electrochemical workstation. The electrochemical system was a standard three-electrode system. The samples were the working electrode and $\mathrm{AgCl}$ electrode was the reference electrode, and platinum electrode was the auxiliary electrode. Before the experiment, the samples were polished and cleaned using alcohol ultrasonic. $\mathrm{NaCl}$ solution with mass fraction of $3.5 \%$ was used during the experiment. The sample should be kept in 3600 s before the test. The alternating current impedance and corrosion rate (Tafel curve) were tested after the open-circuit potential stabilized.

Hitachi SU8010 field emission scanning electron microscope (SEM) (The origin is Tokyo, Japan, purchased from Hitachi High Technologies Corporation) was used to observe the microstructure and electrochemical corrosion morphology. The energy dispersive spectrometer (EDS) was used to analyze element distribution. The samples were ground to 2000\# with $\mathrm{SiC}$ sandpaper, and then mechanical polished. X-ray diffractometer (D8 Advance) (The origin is Germany and purchased from Bruker, Siegsdorf, Germany) was used to assess the crystal structure and for phase analysis. The scanning step was set as $4^{\circ} / \mathrm{min}$ and the diffraction angle $2 \theta$ was selected between $20^{\circ}$ and $100^{\circ}$.

\section{Results and Discussion}

X-ray was used for phase analysis of the three high entropy alloys and the results are shown in Figure 1. Two kinds of BCC structures were identified and labeled BCC1 and BCC2. It can be seen that the position of the diffraction peak changed after the addition of Mo and Sn elements, but the crystal structure did not change, which was attributed to the lattice distortion.

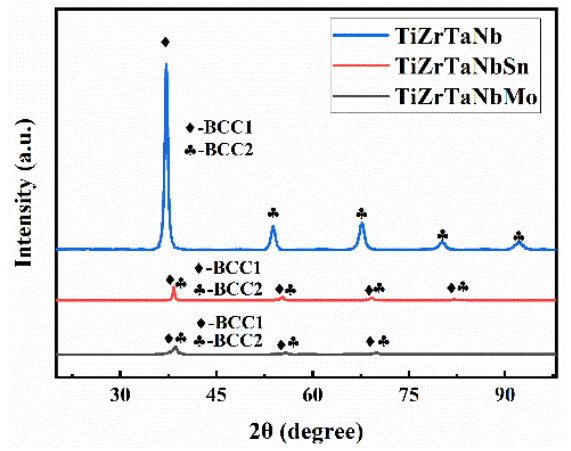

Figure 1. XRD patterns of high entropy alloys.

The microstructure and element distribution of different high entropy alloys were analyzed by SEM and EDS as shown in Figure 2. The microstructure showed a bright region and dark region in BSE mode corresponding to the dendrite and interdendritic, which resulted from the element segregation. $\mathrm{The} \mathrm{Ti}, \mathrm{Zr}, \mathrm{Nb}$ elements were mainly segregated as interdendritic and the Ta element segregated as dendrite. The distribution of $\mathrm{Ti}, \mathrm{Zr}$, 
$\mathrm{Nb}$, Ta in TiZrTaNbMo alloy was similar with TiZrTaNb alloy, while the Mo element was distributed as dendrite as shown in Figure 2c,d. In the TiZrTaNbSn alloy, the Sn element were enriched in interdendritic, while the $\mathrm{Nb}$ element was distributed homogeneously comparing to the TiZrTaNbMo alloy as shown in Figure 2e,f. It can be concluded that $\mathrm{Ta}$, Mo elements are mainly enriched in dendrite and $\mathrm{Zr}, \mathrm{Ti}, \mathrm{Nb}$, Sn elements are mainly distributed in the interdendritic in high-entropy alloys, which is attributed to the different equilibrium distribution coefficients.

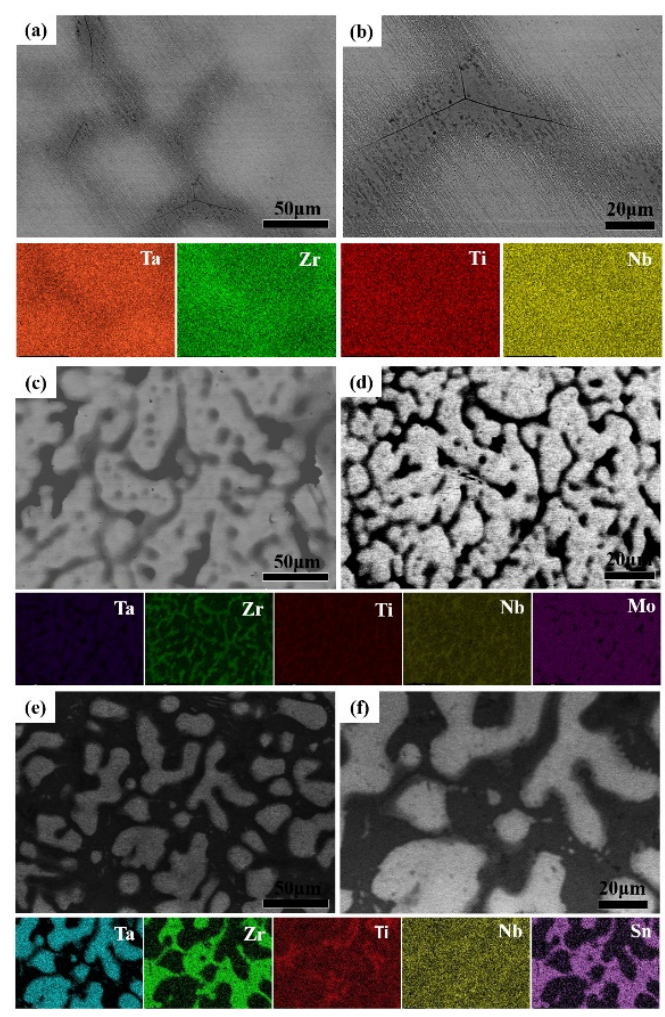

Figure 2. BSE images and corresponding EDS of high entropy alloys: (a,b) TiZrTaNb alloy; (c,d) TiZrTaNbMo alloy; (e,f) TiZrTaNbSn alloy.

In order to study the influence of alloying elements on the corrosion resistance of high entropy alloys, the electrochemical corrosion tests were carried out and the results are shown in Figure 3. The electrochemical impedance can be used to describe the corrosion resistance, and the higher impedance value represents the better corrosion resistance. The electrochemical impedance spectra of high-entropy alloys in $3.5 \% \mathrm{NaCl}$ solution are shown in Figure 3a. The radius of the capacitive reactance arc decreases significantly after the addition of Mo and Sn elements. It can be seen from Figure 3a, TiZrTaNbMo showed the best corrosion resistance, while TiZrTaNbSn showed the worst corrosion resistance among the three high-entropy alloys. The potentiodynamic polarization curves of the three high entropy alloys were similar and the redox reactions occurred as shown in Figure 3b. Anodic polarization is an activation-passivation process, and the electrochemical corrosion parameters are calculated according to Figure $3 \mathrm{~b}$ listed in Table 1 . At the beginning of corrosion, the current density is called $\mathrm{I}_{\text {corr, }}$ which can reveal the corrosion resistance. The corrosion resistance increased with the decrease of $\mathrm{I}_{\text {corr }}$, then it can be concluded that the TiZrNbTaMo alloy has the best corrosion performance. The corrosion potential $\varphi_{\text {corr }}$ can reflect the thermodynamic properties and surface state of the electrode. The corrosion tendency increased with the decrease of $\varphi_{\text {corr }}$, as a result, the corrosion tendency of TiZrTaNbMo alloy was lower than that of TiZrTaNb and TiZrTaNbSn alloys. The passivation current density $\mathrm{I}_{\text {pass }}$ can be used to evaluate the state of corroded system, and the activated state changes to a passivated state depending on the $\mathrm{I}_{\text {pass. }}$. The smaller $\mathrm{I}_{\text {pass }}$ indicates that the system is easier to keep the passivation state. It indicates that the alloys 
have entered the passivation stage, and a protective passivation film is formed on the surface. The $\mathrm{I}_{\text {pass }}$ of TiZrTaNb, TiZrTaNbMo and TiZrTaNbSn alloys were $4.12 \times 10^{-2} \mathrm{a} \cdot \mathrm{cm}^{-2}$, $1.02 \times 10^{-3} \mathrm{a} \cdot \mathrm{cm}^{-2}$ and $5.2 \times 10^{-2} \mathrm{a} \cdot \mathrm{cm}^{-2}$ respectively. The TiZrTaNiMo alloy showed the best corrosion resistance according the corrosion parameters listed in Table 1.
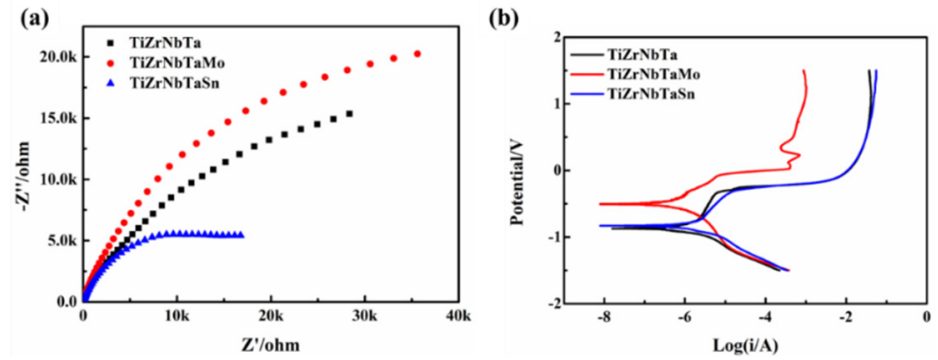

Figure 3. (a) Nyquist diagram and (b) corresponding polarization curves of high entropy alloys.

Table 1. Parameters of electrochemical corrosion of high entropy alloy.

\begin{tabular}{cccc}
\hline Alloy & Icorr/A $\cdot \mathbf{c m}^{-2}$ & $\varphi$ corr/V & Ipass/A.cm $^{-2}$ \\
\hline TiZrTaNb & $7.08 \times 10^{-7}$ & -0.87 & $4.12 \times 10^{-2}$ \\
TiZrTaNbMo & $5.5 \times 10^{-7}$ & -0.499 & $1.02 \times 10^{-3}$ \\
TiZrTaNbSn & $7.87 \times 10^{-7}$ & -0.828 & $5.2 \times 10^{-2}$ \\
\hline
\end{tabular}

The surface morphology of the high entropy alloys after electrochemical corrosion is shown in Figure 4. The surface was etched after electrochemical corrosion for $60 \mathrm{~min}$. The electrochemical corrosion behavior of the high entropy alloys was basically the same, the corrosion mainly occurred at the grain boundary due to interdendritic segregation. The corrosion degree of the three high entropy alloys was different from each other, which corresponds to different corrosion properties. A small number of corrosion particles were attached to the surface of TiZrTaNbMo alloy, and no obvious corrosion was observed, denoting excellent corrosion resistance as shown in Figure $4 \mathrm{~b}$. Severe corrosion occured on the surface of TiZrTaNbSn alloy, and there was a large number of corrosion pits and cracks on the surface as shown in Figure 4c. The deteriorative corrosion property of TiZrTaNbSn alloy was attributed to the interdendritic segregation of the Sn element. The corrosion property of TiZrTaNbSn alloy could be improved by eliminating segregation and further work will be carried out.
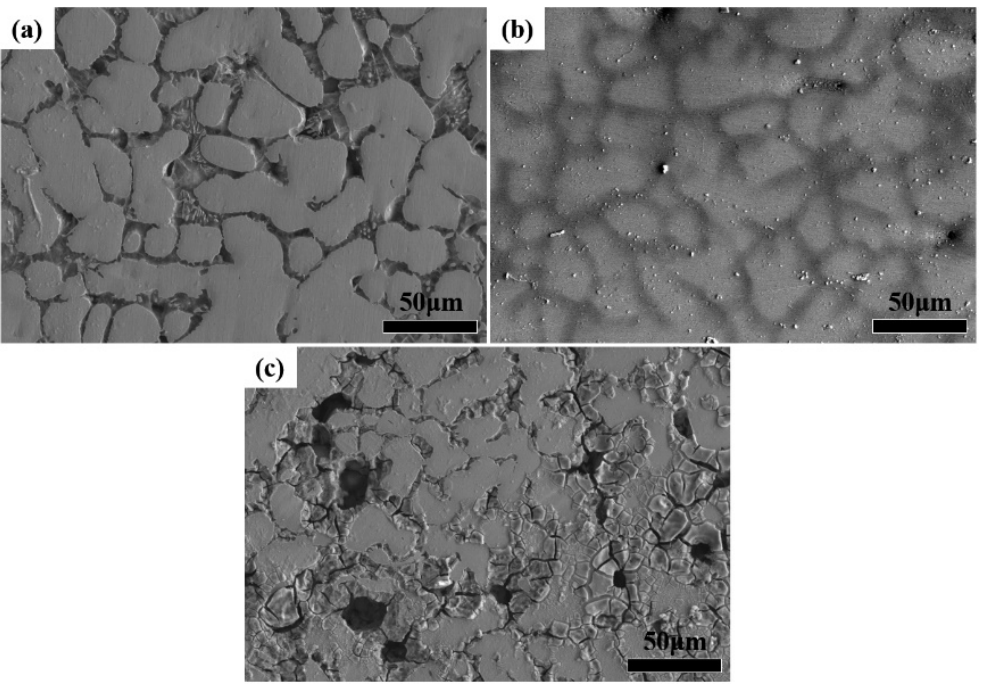

Figure 4. Surface morphologies of high entropy alloys after electrochemical etching: (a) TiZrTaNb alloy; (b) TiZrTaNbMo alloy; (c) TiZrTaNbSn alloy. 


\section{Conclusions}

This paper systematically studied the effect of Mo and Sn elements on the microstructure and corrosion properties of TiZrTaNb alloy, and the main conclusions are as follows:

1. The microstructure of TiZrTaNb, TiZrTaNbMo and TiZrTaNbSn alloys is dendrite with single BCC structures. The addition of Mo and Sn elements promotes dendrite growth and interdendritic segregation.

2. The $\mathrm{Ta}$, Mo elements are mainly enriched in dendrite and $\mathrm{Zr}, \mathrm{Ti}, \mathrm{Nb}$, Sn elements are mainly distributed in the interdendritic in high entropy alloys, which is attributed to the different equilibrium distribution coefficients.

3. The TiZrTaNiMo alloy shows excellent corrosion property according to the corrosion parameters $\mathrm{I}_{\text {corr }}, \varphi_{\text {corr }}, \mathrm{I}_{\text {pass. }}$. The corrosion property of the TiZrTaNb alloy deteriorated after the addition of $\mathrm{Sn}$ element and this is attributed to the interdendritic segregation of Sn element.

Author Contributions: Conceptualization, T.M.; methodology, Q.L., Y.J. and D.D.; formal analysis, D.Z.; investigation, X.W., Y.J. and Q.L.; resources, D.Z., and T.M.; data curation, Q.L.; writingoriginal draft preparation, Q.L.; writing — review and editing, D.Z. and T.M. All authors have read and agreed to the published version of the manuscript.

Funding: The work was supported by National Natural Science of Foundation of China (Grant No. 52001262, 52071188, 52171120), Zhejiang Province Natural Science Foundation of China (Grant No.: ZY22E010293, LQ20E010003).

Institutional Review Board Statement: Not applicable.

Informed Consent Statement: Informed consent was obtained from all subjects involved in the study.

Data Availability Statement: Not applicable.

Conflicts of Interest: The authors declare no conflict of interest.

\section{References}

1. Zhang, Y.; Zuo, T.T.; Tang, Z.; Gao, M.C.; Dahmen, K.A.; Liaw, P.K.; Lu, Z.P. Microstructures and properties of high-entropy alloys. Prog. Mater. Sci. 2014, 61, 1-93. [CrossRef]

2. He, M.Y.; Shen, Y.F.; Jia, N.; Liaw, P.K. C and N doping in high-entropy alloys: A pathway to achieve desired strength-ductility synergy. Appl. Mater. Today 2021, 25, 101162. [CrossRef]

3. Krishna, Y.V.; Jaiswal, U.K.; Rahul, M.R. Machine learning approach to predict new multiphase high entropy alloys. Scr. Mater. 2021, 197, 113804. [CrossRef]

4. Zhou, N.X.; Jiang, S.C.; Huang, T.; Qin, M.D.; Hu, T.; Luo, J. Single-phase high-entropy intermetallic compounds (HEICs): Bridging high-entropy alloys and ceramics. Sci. Bull. 2019, 64, 856-864. [CrossRef]

5. Long, Y.; Li, G.Q.; Liang, X.B.; Peng, H.Y. Fine-grained FeCoNi(CuAl)(x) high entropy alloys: Phase transformation, microstructure evolution and mechanical properties. Front. Mater. 2020, 7, 537812. [CrossRef]

6. Ma, Y.J.; Ma, Y.; Wang, Q.S.; Schweidler, S.; Botros, M.; Fu, T.T.; Hahn, H.; Brezesinski, T.; Breitung, B. High-entropy energy materials: Challenges and new opportunities. Energy Environ. Sci. 2021, 14, 2883-2905. [CrossRef]

7. Chuang, M.H.; Tsai, M.H.; Wang, W.R.; Lin, S.J; Yeh, J.W. Microstructure and wear behavior of AlxCo1.5CrFeNi1.5Ti high-entropy alloys. Acta Mater. 2011, 59, 6308-6317. [CrossRef]

8. Nong, Z.S.; Li, D.S.; Zhu, J.C.; Yu, H.L.; Lai, Z.H. Effect of aluminum content on microstructure and wear resistance of CuCrFeMnTiAlx high-entropy alloy. Rare Met. Mater. Eng. 2011, 40, 550-554.

9. Xiao, J.K.; Tan, H.; Chen, J.; Martini, A.; Zhang, C. Effect of carbon content on microstructure, hardness and wear resistance of CoCrFeMnNiCx high-entropy alloys. J. Alloy. Compd. 2020, 847, 156533. [CrossRef]

10. Yin, B.L.; Curtin, W.A. Origin of high strength in the CoCrFeNiPd high-entropy alloy. Mater. Res. Lett. 2020, 8, 209-215. [CrossRef]

11. Shang, C.Y.; Axinte, E.; Ge, W.J.; Zhang, Z.T.; Wang, Y. High-entropy alloy coatings with excellent mechanical, corrosion resistance and magnetic properties prepared by mechanical alloying and hot pressing sintering. Surf. Interfaces 2017, 9, 36-43. [CrossRef]

12. Wang, S.P.; Xu, J. (TiZrNbTa)-Mo high-entropy alloys: Dependence of microstructure and mechanical properties on Mo concentration and modeling of solid solution strengthening. Intermetallics 2018, 95, 59-72. [CrossRef]

13. Eletia, R.R.; Stepanova, N.; Yurchenko, N.; Zherebtsov, S.; Marescab, F. Cross-kink unpinning controls the medium- to hightemperature strength of body-centered cubic NbTiZr medium-entropy alloy. Scr. Mater. 2021, 209, 114367. [CrossRef]

14. Eleti, R.R.; Stepanov, N.; Yurchenko, N.; Klimenko, D.; Zherebtsov, S. Plastic deformation of solid-solution strengthened Hf-Nb-Ta-Ti-Zr body-centered cubic medium/high-entropy alloys. Scr. Mater. 2021, 200, 113927. [CrossRef] 
15. Nguyen, V.T.; Qian, M.; Shi, Z.; Song, T.; Huang, L.; Zou, J. A novel quaternary equiatomic Ti-Zr-Nb-Ta medium entropy alloy (MEA). Intermetallics 2018, 101, 39-43. [CrossRef]

16. Liao, M.Q.; Liu, Y.; Min, L.J.; Lai, Z.H.; Han, T.Y.; Yang, D.N.; Zhu, J.C. Alloying effect on phase stability, elastic and thermodynamic properties of $\mathrm{Nb}-\mathrm{Ti}-\mathrm{V}-\mathrm{Zr}$ high entropy alloy. Intermetallics 2018, 101, 152-164. [CrossRef]

17. Todai, M. Novel TiNbTaZrMo high-entropy alloys for metallic biomaterials. Scr. Mater. 2017, 129, 65-68. [CrossRef]

18. Raabe, D.; Tasan, C.C.; Springer, H.; Bausch, M. From high-entropy alloys to high-entropy steels. Steel Res. Int. 2015, 86, 1127-1138. [CrossRef]

19. Raza, A.; Abdulahad, S.; Kang, B.; Ryu, H.J.; Hong, S.H. Corrosion resistance of weight reduced AlxCrFeMoV high entropy alloys. Appl. Surf. Sci. 2019, 485, 368-374. [CrossRef]

20. Shang, C.Y.; Axinte, E.; Sun, J.; Li, X.T.; Li, P.; Du, J.W.; Qiao, P.C.; Wang, Y. CoCrFeNi(W1-xMox) high-entropy alloy coatings with excellent mechanical properties and corrosion resistance prepared by mechanical alloying and hot pressing sintering. Mater. Des. 2017, 117, 193-202. [CrossRef]

21. Li, J.J.; Zhao, X.Y.; Hu, L.N.; Ma, L.Q.; Shen, X.D. Electrochemical properties of Co-S/x wt.\% AB(5) composite materials. Sci. China-Technol. Sci. 2015, 58, 1355-1359. [CrossRef] 